\title{
BLOW UP, GLOBAL EXISTENCE AND GROWTH RATE \\ ESTIMATES IN NONLINEAR PARABOLIC SYSTEMS
}

BY

\section{JOANNA RENCŁAWOWICZ (WARSZAWA)}

Abstract. We prove Fujita-type global existence and nonexistence theorems for a system of $m$ equations $(m>1)$ with different diffusion coefficients, i.e.

$$
u_{i t}-d_{i} \Delta u_{i}=\prod_{k=1}^{m} u_{k}^{p_{k}^{i}}, \quad i=1, \ldots, m, x \in \mathbb{R}^{N}, t>0,
$$

with nonnegative, bounded, continuous initial values and $p_{k}^{i} \geq 0, i, k=1, \ldots, m, d_{i}>0$, $i=1, \ldots, m$. For solutions which blow up at $t=T<\infty$, we derive the following bounds on the blow up rate:

$$
u_{i}(x, t) \leq C(T-t)^{-\alpha_{i}}
$$

with $C>0$ and $\alpha_{i}$ defined in terms of $p_{k}^{i}$.

1. Introduction. We consider the following semilinear problem:

$$
u_{i t}-d_{i} \Delta u_{i}=\prod_{k=1}^{m} u_{k}^{p_{k}^{i}}, \quad i=1, \ldots, m,
$$

for $x \in \mathbb{R}^{N}, t>0$ and

$$
u_{i}(0, x)=u_{0 i}(x), \quad i=1, \ldots, m, x \in \mathbb{R}^{N},
$$

where $d_{i}, p_{k}^{i}, i, k=1, \ldots, m$, are nonnegative constants and $u_{0 i}, i=1, \ldots, m$, are nonnegative, continuous, bounded functions, $N, m \geq 1$.

As the main results, we present a classification of solutions according to their time existence, and bounds on the rate of blow up for nonglobal solutions. It turns out that blow up is driven by the nonlinearity in our system, i.e. $\left(u_{1}, \ldots, u_{m}\right)$ blows up at the same rate as the solutions of the corresponding kinetic system. Namely, if $T$ denotes the maximal existence time of $u$, we prove that

$$
u_{i}(x, t) \leq C(T-t)^{-\alpha_{i}}, \quad i=1, \ldots, m,
$$

where the $\alpha_{i}$ are given in terms of $p_{k}^{i}$.

2000 Mathematics Subject Classification: 35B30, 35B50, 35K55, 35K57.

Key words and phrases: blow up, global existence, reaction-diffusion system, invariant region, invariant manifold. 
We briefly review some related work. The system (1.1) for $m=1$ or $m=2$ and $d_{i}=1$ has been analyzed by several authors. We mention the papers of Fujita ([Fu1], [Fu2]) for the scalar problem, Lu [L] and Escobedo and Levine $[\mathrm{EL}]$ for a system of two equations. Some special problems, namely (1.1) with $p_{k}^{i}=0$ for $k \neq i+1, i=1, \ldots, m-1$ and $p_{k}^{m}=0$ for $k \neq 1$ (the so-called "completely coupled systems"), have been considered by Escobedo and Herrero [EH] for $m=2$ and by the author for $m \geq 3$ [R1], [R4]. The system (1.1) for $m=2$ and positive $d_{i}$ is examined in [R2] where the result analogous to Theorem 2 below is proved. In [R3] the system (1.1) with $m=3$ and $d_{i}=1, i=1,2,3$, is treated and the assertions corresponding to Theorem 2 and Theorem 1B below are shown. To our knowledge, the paper $[\mathrm{AHV}]$ is the only work where the estimates of the rate of the growth of solutions for a completely coupled system of two equations in $\mathbb{R}^{N} \times(0, T)$, namely for

$$
\begin{aligned}
& u_{t}=\Delta u+v^{p} \\
& v_{t}=\Delta v+u^{q},
\end{aligned}
$$

have been proved. However, the method used, based on classical regularity techniques, seems to be suitable only for the system (1.2). We also mention $[\mathrm{CM}]$ where the authors prove similar bounds, but only for some class of radially symmetric solutions, eliminating any possibility of oscillation in time. In our work we use an idea originally due to $\mathrm{Lu}[\mathrm{L}]$, based on the comparison principles and the concept of invariant region. It is worth noticing that these tools are crucial to derive our growth rate estimates. We also apply the results of $[\mathrm{LS}]$ to construct a subsolution to our system.

For the statement of the main results, we need some notations. Let

$$
A_{m}=\left[p_{k}^{i}\right], \quad i, k=1, \ldots, m,
$$

where $i$ labels the rows and $k$ the columns of the matrix. It is clear that, for definiteness, we can assume henceforth

$$
\min _{i} \sum_{k=1}^{m} p_{k}^{i}=\sum_{k=1}^{m} p_{k}^{1} .
$$

By $\alpha=\left(\alpha_{1}, \ldots, \alpha_{m}\right)$ we denote the unique solution of

$$
\left(A_{m}-I\right) \alpha^{t}=(1, \ldots, 1)^{t} .
$$

We put

$$
\delta=\operatorname{det}\left(A_{m}-I\right) .
$$

Denoting by $D_{k}\left(A_{m}-I\right)$ the matrix $A_{m}-I$ with column $k$ replaced by the vector $(1, \ldots, 1)^{t}$, we have, whenever $\delta \neq 0$,

$$
\alpha_{k}=\delta^{-1} \operatorname{det}\left(D_{k}\left(A_{m}-I\right)\right), \quad k=1, \ldots, m .
$$


We also set

$$
b_{k}=\alpha_{k} / \alpha_{1}, \quad k=1, \ldots, m,
$$

assuming that $\alpha_{1} \neq 0, b_{k}>0$. We put

$$
r=\sum_{k=1}^{m} b_{k} p_{k}^{1}=\frac{1+\alpha_{1}}{\alpha_{1}} .
$$

REMARK 1.1. Without loss of generality, we can assume that $b_{k}<1$ for $k=2, \ldots, m$, which implies $\max _{k} \alpha_{k}=\alpha_{1}$. Otherwise, we have $\max _{k} \alpha_{k}$ $=\alpha_{j}$ and instead of $b_{k}, r$ we define $b_{k}(j)=\alpha_{k} / \alpha_{j}, r(j)=\sum_{k=1}^{m} b_{k}(j) p_{k}^{j}=$ $\left(1+\alpha_{j}\right) / \alpha_{j}$ where $b_{k}(j)<1$ for $k=1, \ldots, m, k \neq j$.

By the above remark we set $r=r(j)$ and formulate our results.

THEOREM 1. Assume that $p_{1}^{1}>1$.

A. If $\left(\sum_{k=1}^{m} p_{k}^{1}-1\right)^{-1}<N / 2$, then for sufficiently small initial data the solutions of (1.1) exist globally whereas all solutions with initial values large enough blow up in finite time.

B. If $\left(\sum_{k=1}^{m} p_{k}^{1}-1\right)^{-1} \geq N / 2$, then every nontrivial solution of (1.1) is nonglobal.

THEOREM 2. Assume that $p_{1}^{1} \leq 1$ and $\delta \neq 0$.

A. If $\max _{k} \alpha_{k}<0$ (i.e. $\left.0 \leq r<1\right)$, then all solutions of (1.1) are global.

B. If $0<\max _{k} \alpha_{k}<N / 2$ (i.e. $r>1+2 / N$ ), then there are both nontrivial global solutions and nonglobal solutions of (1.1).

C. If $\min _{k} \alpha_{k} \geq N / 2$ (i.e. $1<r \leq 1+2 / N$ with $b_{k}>1$ for $k=2, \ldots, m$ ), then all nontrivial solutions of (1.1) are nonglobal.

THEOREM 3. Let $u$ be a solution of (1.1) which blows up at $x_{0}$ and $T<\infty$. Then for any compact subset $\Omega \ni x_{0}$ there exists a constant $C>0$ such that the following bounds hold:

$$
\max _{x \in \Omega} u_{i}(x, t) \geq C(T-t)^{-\alpha_{i}}, \quad i=1, \ldots, m .
$$

THEOREM 4. Let $u$ be a solution of (1.1) in $\mathbb{R}^{N} \times(0, T)$. Assume that $\max _{i} \alpha_{i}=\alpha_{1}$ and $\min _{i} \alpha_{i}>0$. If one of the following conditions holds:

$$
\begin{gathered}
N=1,2 \quad \text { or } \quad N \geq 3, \alpha_{1} \geq \frac{N-2}{4}, \\
d_{1} \Delta u_{01}+A_{1} u_{01}^{1+1 / \alpha_{1}}>0,
\end{gathered}
$$

then for some constant $C>0$,

$$
u_{i}(x, t) \leq C(T-t)^{-\alpha_{i}}, \quad i=1, \ldots, m
$$

Some auxiliary assertions are gathered in the next section. The proofs of the global existence and blow up results in the case $p_{1}^{1}>1$ can be found 
in Section 3, whereas the contrary situation is discussed in Section 4. The lower and upper bounds on the blow up rate are proved in Section 5 .

2. Preliminary results. Let $S_{i}(t)$ denote the semigroup operator for the heat equation with diffusion coefficient $d_{i}$, i.e.

$$
S_{i}(t) w_{0}(x)=\int_{\mathbb{R}^{N}}\left(4 d_{i} \pi t\right)^{-N / 2} \exp \left(-\frac{|x-y|^{2}}{4 d_{i} t}\right) w_{0}(y) d y .
$$

We consider classical nonnegative solutions of (1.1). Such solutions satisfy

$$
u_{i}(t)=S_{i}(t) u_{0 i}+\int_{0}^{t} S_{i}(t-s) \prod_{k=1}^{m}\left(u_{k}(s)\right)^{p_{k}^{i}} d s, \quad i=1, \ldots, m .
$$

In particular, we have

$$
\begin{aligned}
u_{i}(\tau) & =S_{i}\left(\tau-t_{i}\right) u_{i}\left(t_{i}\right)+\int_{0}^{\tau-t_{i}} S_{i}\left(\tau-t_{i}-s\right) \prod_{k=1}^{m}\left(u_{k}(s)\right)^{p_{k}^{i}} d s \\
& \geq S_{i}\left(\tau-t_{i}\right) u_{i}\left(t_{i}\right), \quad i=1, \ldots, m .
\end{aligned}
$$

Let $u$ be a nondegenerate, nonnegative solution of (1.1), i.e. no component vanishes identically on $\mathbb{R}^{N} \times(0, T)$. If $\left(x_{i}, t_{i}\right)$ are such that $u_{i}\left(x_{i}, t_{i}\right)>0$, $i=1, \ldots, m$, then in view of the positivity of $S_{i}(t)$ the above variation of constants formula implies that $u_{i}(\tau)>0$ for $\tau>t_{i}$. Consequently, $u_{i}(x, \tau)>0$ for $x \in \mathbb{R}^{N}, \tau>t_{*}=\max _{i=1, \ldots, m} t_{i}$.

LEMMA 2.1. Let $u=\left(u_{1}, \ldots, u_{m}\right)$ be a nondegenerate solution of (1.1). Then we can choose $\tau=\tau\left(u_{01}, \ldots, u_{0 m}\right)$ and some constants $c>0$ and $a>0$ such that $\min u_{i}(\tau) \geq c e^{-a|x|^{2}}$.

Proof. We know that there exists $t_{0}$ such that $u_{i}(x, \tau)>0$ for $\tau>t_{0}$. Thus, if necessary, we can shift the initial time from zero to some $t>t_{0}$ to obtain the positivity of the initial data. We can assume that for some $R>0$,

$$
\nu_{i}=\inf \left\{u_{0 i}(x):|x|<R\right\}>0 .
$$

Using (2.2) we have, by positivity of $u_{k}$,

$$
u_{i}(t) \geq S_{i}(t) u_{0 i} \geq \nu_{i}\left(4 d_{i} \pi t\right)^{-N / 2} \exp \left(\frac{-|x|^{2}}{4 d_{i} t}\right) \int_{|y| \leq R} \exp \left(\frac{-|y|^{2}}{4 d_{i} t}\right) d y .
$$

We define

$$
\begin{gathered}
\bar{u}_{i}(t)=u_{i}\left(t+\tau_{0}\right) \quad \text { for some } \tau_{0}>0 \\
a_{i}=\frac{1}{4 d_{i} \tau_{0}}, \quad c_{i}=\nu_{i}\left(4 d_{i} \pi \tau_{0}\right)^{-N / 2} \int_{|y| \leq R} \exp \left(\frac{-|y|^{2}}{4 d_{i} \tau_{0}}\right) d y .
\end{gathered}
$$


Then

$$
\bar{u}_{i}(0)=u_{i}\left(\tau_{0}\right)>c_{i} \exp \left(-a_{i}|x|^{2}\right)
$$

To get the assertion, we choose $a, c, \tau_{0}$ suitable for all $u_{i}$.

We introduce the following kinetic system, corresponding to (1.1):

$$
\left\{\begin{array}{l}
u_{i}^{\prime}=\prod_{k=1}^{m} u_{k}^{p_{k}^{i}}, \quad i=1, \ldots, m \\
u_{i}(0)=u_{0 i} .
\end{array}\right.
$$

Definition 2.2 ([H], Definition 6.1.1). A set $D \subset \mathbb{R} \times \mathbb{R}^{N}$ is an invariant manifold for an equation $d u / d t+A u=f(t, u)$ provided for any $\left(t_{0}, u_{0}\right) \in D$, there exists a solution $u$ of the equation on an interval containing $t_{0}$ with $u\left(t_{0}\right)=u_{0}$ and $(t, u(t)) \in D$ on this interval.

LEMMA 2.3. The set

$$
\begin{aligned}
& \partial M=\left\{\left(u_{1}, \ldots, u_{m}\right) \mid F\left(u_{1}, \ldots, u_{m}\right)=\left(F_{1}, \ldots, F_{m-1}\right)=\Theta ;\right. \\
& \left.F_{j}\left(u_{1}, \ldots, u_{m}\right)=u_{j+1}-a_{j+1} u_{j}^{b_{j+1} / b_{j}}, j=1, \ldots, m-1 ; u_{i} \geq 0\right\},
\end{aligned}
$$

where $\Theta=(0, \ldots, 0)$ and $a_{j}$ are constants given by the conditions

$$
a_{1}=1, \quad b_{i} \prod_{j=1}^{i} a_{j}^{b_{i} / b_{j}}=\prod_{k=1}^{m}\left(\prod_{j=1}^{k} a_{j}^{b_{k} / b_{j}}\right)^{p_{k}^{i}}, \quad i=2, \ldots, m,
$$

is an invariant manifold for (2.3).

Pr o of. Notice that along the set $F=\Theta$ we have

$$
u_{k}=a_{k} u_{k-1}^{b_{k} / b_{k-1}}=\prod_{j=1}^{k} a_{j}^{b_{k} / b_{j}} u_{1}^{b_{k}} .
$$

Computing the derivative of $F_{i}, i=1, \ldots, m-1$, with respect to $t$ and using (2.3) and (2.5) we have

$$
\begin{aligned}
\left.\frac{\partial F_{i}}{\partial t}\left(u_{1}, \ldots, u_{m}\right)\right|_{F=\Theta} & u_{i+1}^{\prime}-\left.a_{i+1} \frac{b_{i+1}}{b_{i}} u_{i}^{b_{i+1} / b_{i}-1} u_{i}^{\prime}\right|_{F=\Theta} \\
= & \prod_{k=1}^{m} u_{k}^{p_{k}^{i+1}}-\left.a_{i+1} \frac{b_{i+1}}{b_{i}} u_{i}^{b_{i+1} / b_{i}-1} \prod_{k=1}^{m} u_{k}^{p_{k}^{i}}\right|_{F=\Theta} \\
= & \prod_{k=1}^{m}\left(\prod_{j=1}^{k} a_{j}^{b_{k} / b_{j}} u_{1}^{b_{k}}\right)^{p_{k}^{i+1}}-a_{i+1} \frac{b_{i+1}}{b_{i}}\left(\prod_{j=1}^{i} a_{j}^{b_{i} / b_{j}} u_{1}^{b_{i}}\right)^{b_{i+1} / b_{i}-1} \\
& \times \prod_{k=1}^{m}\left(\prod_{j=1}^{k} a_{j}^{b_{k} / b_{j}} u_{1}^{b_{k}}\right)^{p_{k}^{i}} .
\end{aligned}
$$


By (1.5)-(1.6), we check

$$
\sum_{k=1}^{m} b_{k} p_{k}^{i+1}=\frac{1+\alpha_{i+1}}{\alpha_{1}}=\frac{1+\alpha_{i}}{\alpha_{1}}+\frac{\alpha_{i+1}-\alpha_{i}}{\alpha_{1}}=\sum_{k=1}^{m} b_{k} p_{k}^{i}+b_{i+1}-b_{i}
$$

Therefore, using (2.4) we get

$$
\left.\frac{\partial F_{i}}{\partial t}\left(u_{1}, \ldots, u_{m}\right)\right|_{F=\Theta}=0 \text {. }
$$

LEMMA 2.4. (i) If $r \leq 1$, then any nonnegative solution of (2.3) exists globally.

(ii) If $r>1$, then any nonnegative nontrivial solution of (2.3) blows up in finite time.

Proof. First, we take $u_{0} \in \partial M$, i.e. $u_{0(i+1)}=a_{i+1} u_{0 i}, i=1, \ldots, m-1$, $u_{01} \geq 0$. By Lemma 2.3, $\partial M$ is an invariant manifold for the system (2.3), so $u(t)=\left(u_{1}(t), \ldots, u_{m}(t)\right) \in \partial M$, because $u(0) \in \partial M$. This means that $u_{i+1}(t)=a_{i+1}\left[u_{i}(t)\right]^{b_{i+1} / b_{i}}$ for any $t \in[0, T)$. Hence, system (2.3) on $\partial M$ reduces to the scalar equation

$$
u_{1}^{\prime}=a u_{1}^{r}, \quad u_{1}(0)=u_{01}>0,
$$

where $a^{1 / r}=\prod_{k=1}^{m}\left(\prod_{j=1}^{k} a_{j}^{1 / b_{j}}\right)$.

We can easily obtain the solution $u_{1}$ of this equation by integrating, and the conclusions (i) and (ii) hold on $\partial M$.

Next, we assume $u_{0} \notin \partial M$. We consider two cases:

a) $r \leq 1$. Then we choose $\bar{u}_{01}$ in such a way that

$$
0 \leq u_{0 i} \leq\left(\prod_{j=1}^{i} a_{j}^{1 / b_{j}} \bar{u}_{01}\right)^{b_{i}}=\bar{u}_{0 i}, \quad i=1, \ldots, m
$$

By (2.5) we get $\bar{u}_{0(i+1)}=a_{i+1} \bar{u}_{0 i}$ for $i=1, \ldots, m-1$, therefore $\bar{u}_{0}=$ $\left(\bar{u}_{01}, \ldots, \bar{u}_{0 m}\right) \in \partial M$. Then for the supersolution $\bar{u}=\left(\bar{u}_{1}, \ldots, \bar{u}_{m}\right)$ of $(2.3)$ we can apply Lemma 2.3 to infer (i). Moreover, the comparison principle (see [LS], Theorem 1.2) gives

$$
0 \leq u(t) \leq \bar{u}(t)
$$

whence the conclusion (i) holds for $u(t)$.

b) $r>1$. Then by the Lipschitz condition we have uniqueness, so $u_{0} \neq 0$ implies $u(t)>0, t \geq t_{0}$. By this condition we can assume $u_{0}>0$ and take $\underline{u}_{01}$ such that

$$
0<\underline{u}_{0 i}=\left(\prod_{j=1}^{i} a_{j}^{1 / b_{j}} \underline{u}_{01}\right)^{b_{i}} \leq u_{0 i}, \quad i=1, \ldots, m
$$


Since $\underline{u}_{0(i+1)}=a_{i+1} \underline{u}_{0 i}^{b_{i+1} / b_{i}}$, it follows that $\underline{u}(t) \in \partial M$ (by Lemma 2.3) and for the subsolution $\underline{u}(t)$ to $(2.3)$ we obtain part (ii). The same property remains true for $u(t)$ by the comparison theorem, i.e. $0 \leq \underline{u}(t) \leq u(t)$. Thus the proof is complete.

Definition 2.5. Let $d u / d t+A u=f(t, u), u=\left(u_{1}, \ldots, u_{m}\right)$. A region $D$ is called a regular invariant region for this system if the conditions $u_{0} \in C^{2}$, $u_{0} \in \partial D$ imply that $(t, u(x, t)) \in D$ (where $u$ denotes a solution of this system).

LEMMA 2.6. Let $u=\left(u_{1}, \ldots, u_{m}\right), F(u)=\left(F_{1}(u), \ldots, F_{m-1}(u)\right)$, and

$$
\begin{gathered}
F_{i}(u)=u_{i+1}-a_{i+1} u_{i}^{b_{i+1} / b_{i}}, \quad i=1, \ldots, m-1 \\
M=\left\{u \mid F(u) \leq \Theta, u_{i} \geq 0,\left(d_{i+1}-d_{i}\right) \Delta u_{0 i} \leq 0\right. \\
\text { for } \left.u_{0 i}=u_{i}(0) \in C^{2}, i=1, \ldots, m\right\} .
\end{gathered}
$$

Then $M$ is a regular invariant region for (1.1) if $b_{i+1} / b_{i} \leq 1, i=1, \ldots, m-1$.

Proof. We assume that $u_{0} \in \partial M, u_{0} \in C^{2}$, i.e. $u_{0(i+1)}=a_{i+1} u_{0 i}^{b_{i+1} / b_{i}}$, $u_{0 i} \geq 0,\left(d_{i+1}-d_{i}\right) \Delta u_{0 i} \leq 0$. Using (2.5) we have

$$
\begin{aligned}
\left.\frac{\partial F_{i}\left(u_{1}(x, t), \ldots, u_{m}(x, t)\right)}{\partial t}\right|_{F=\Theta, t=0} & \\
= & \frac{\partial}{\partial t} u_{i+1}-\left.a_{i+1} \frac{b_{i+1}}{b_{i}} u_{i}^{b_{i+1} / b_{i}-1} \frac{\partial}{\partial t} u_{i}\right|_{F=\Theta, t=0} \\
= & d_{i+1} \Delta u_{0(i+1)}-a_{i+1} \frac{b_{i+1}}{b_{i}} u_{0 i}^{b_{i+1} / b_{i}-1} d_{i} \Delta u_{0 i} \\
& +\left.\left[\prod_{k=1}^{m} u_{0 k}^{p_{k}^{i+1}}-a_{i+1} \frac{b_{i+1}}{b_{i}} u_{0 i}^{b_{i+1} / b_{i}-1} \prod_{k=1}^{m} u_{0 k}^{p_{k}^{i}}\right]\right|_{F=\Theta} \\
= & \left(d_{i+1}-d_{i}\right) a_{i+1} \frac{b_{i+1}}{b_{i}} u_{0 i}^{b_{i+1} / b_{i}-1} \Delta u_{0 i} \\
& +d_{i+1} a_{i+1} \frac{b_{i+1}}{b_{i}}\left(\frac{b_{i+1}}{b_{i}}-1\right) u_{0 i}^{b_{i+1} / b_{i}-2}\left|\nabla u_{0 i}\right|^{2} \leq 0
\end{aligned}
$$

for $i=1, \ldots, m-1$ since $b_{i+1} \leq b_{i}$.

If $\left(d_{i}=d_{j}\right.$ or $\left.\Delta u_{0 i} \equiv 0\right)$ and simultaneously $\nabla u_{0 i} \equiv 0$ then $\partial M$ is a regular invariant manifold for (1.1).

By (2.6) we infer

$$
\left.\frac{\partial F(u(x, t))}{\partial t}\right|_{F=\Theta, t=0} \leq \Theta .
$$

We now proceed to prove that $M$ is locally invariant, i.e. $u(x, t) \in M$ for all $x \in \mathbb{R}^{N}$ and $t$ sufficiently small. 
By (2.5),

$$
\left|\nabla u_{0 i}(x)\right|=b_{i} \prod_{j=1}^{i} a_{j}^{b_{i} / b_{j}}\left(u_{01}(x)\right)^{b_{i}-1}\left|\nabla u_{01}(x)\right| .
$$

Let $x_{0} \in \mathbb{R}^{N}$ and $\left|\nabla u_{01}\left(x_{0}\right)\right|>0$. Then, by (2.6), (2.7) and assuming $b_{i+1}<b_{i}$ we get $F_{t}\left(u\left(x_{0}, 0\right)\right)<\Theta$ (if $b_{i+1}=b_{i}$ we can repeat our considerations taking $y_{0}$ such that $\left.\Delta u_{01}\left(y_{0}\right)>0\right)$.

Since $\left.F\right|_{t=0}=\Theta$, it follows that there exists $\delta_{i}\left(x_{0}\right)$ satisfying

$$
F_{i}\left(u\left(x_{0}, t\right)\right)<0 \quad \text { for } 0<t<\delta_{i}\left(x_{0}\right),
$$

whence

$$
F\left(u\left(x_{0}, t\right)\right)<\Theta \quad \text { for } 0<t<\min _{i} \delta_{i}\left(x_{0}\right) .
$$

If $F_{t}\left(u\left(x_{0}, 0\right)\right)=\Theta$, then we can choose $\varepsilon>0$ and $x^{*}=\left(x_{1}, x_{0,1}, \ldots, x_{0 N}\right)$ such that

$$
\left|\nabla u_{01}\left(x^{*}\right)\right|>0 \quad \text { for } 0<\left|x_{1}-x_{01}\right|<\varepsilon
$$

and then $F_{t}\left(u\left(x^{*}, 0\right)\right)<\Theta$. It follows that for some $\delta\left(x^{*}\right)>0$,

$$
F\left(x^{*}, t\right)<F\left(x^{*}, 0\right)=\Theta \quad \text { for } 0<t<\delta\left(x^{*}\right)
$$

and there exists $\varepsilon\left(x^{*}\right)>0$ such that

$$
F(x, t)<\Theta \quad \text { for }\left|x-x^{*}\right|<\varepsilon\left(x^{*}\right), 0<t<\delta\left(x^{*}\right) .
$$

We note that assuming $F\left(x_{0}, t\right)>\Theta$ we would get a contradiction. Indeed, this condition implies $F(x, t)>\Theta$ for some $x$ in a neighbourhood of $x_{0}$ and this is incompatible with the above result. Thus, we have proved the local invariance of $M$.

Now, we want to verify that $u(x, t) \in M$ for any $x \in \mathbb{R}^{N}$ and $t \in[0, T)$. Assume that this condition is not satisfied, i.e. there exist $t_{0}, x_{0} \in \mathbb{R}^{N}$ such that

$$
F(x, t)<\Theta, \quad x \in \mathbb{R}^{N}, t<t_{0},
$$

and

$$
F\left(x_{0}, t\right)>\Theta, \quad t_{0}<t<t_{0}+\delta_{0}\left(x_{0}\right) .
$$

Take a regular supersolution $\bar{u}(x, t)$ of (1.1) with initial values $\bar{u}\left(x, t_{0}\right)$ such that

$$
\begin{gathered}
\bar{u}_{i+1}\left(x, t_{0}\right)=a_{i+1}\left(\bar{u}_{i}\left(x, t_{0}\right)\right)^{b_{i+1} / b_{i}}, \quad \bar{u}_{1}\left(x, t_{0}\right)=u_{1}\left(x, t_{0}\right), \\
\left(d_{i+1}-d_{i}\right) \Delta \bar{u}_{i}\left(x, t_{0}\right) \leq 0 .
\end{gathered}
$$

Then, since $\bar{u}\left(x, t_{0}\right) \geq u\left(x, t_{0}\right)$, the comparison theorem gives

$$
u(x, t) \leq \bar{u}(x, t), \quad x \in \mathbb{R}^{N}, t \in\left[t_{0}, T^{*}\right) .
$$


Using (2.8), we also obtain

$$
\bar{u}_{i+1}(x, t)>u_{i+1}\left(x_{0}, t\right)>a_{i+1}\left(u_{i}\left(x_{0}, t\right)\right)^{b_{i+1} / b_{i}}
$$

for $i=1, \ldots, m-1, t_{0}<t<t_{0}+\delta_{0}\left(x_{0}\right)$, and by continuity

$$
\bar{u}_{i+1}(x, t)>a_{i+1}\left(u_{i}(x, t)\right)^{b_{i+1} / b_{i}}, \quad\left|x-x_{0}\right|<\eta, t_{0}<t<t_{0}+\delta_{0}\left(x_{0}\right) .
$$

Therefore, putting

$$
\varepsilon_{i}(x, t)=\bar{u}_{i+1}(x, t)-a_{i+1}\left(u_{i}(x, t)\right)^{b_{i+1} / b_{i}}
$$

we have $\varepsilon_{i}(x, t)>0$ for $t_{0}<t<t_{0}+\delta_{0}(x),\left|x-x_{0}\right|<\eta$. We can also choose $\delta_{1}(x)>0$ satisfying

$$
0 \leq a_{2} \bar{u}_{1}^{b_{2}}(x, t)-a_{2} u_{1}^{b_{2}}(x, t)<\frac{1}{2} \varepsilon_{1}(x, t), \quad t_{0}<t<t_{0}+\delta_{1}(x) .
$$

Let $\delta(x)=\min \left\{\delta_{0}(x), \delta_{1}(x)\right\}$. Then

$$
\varepsilon_{1}(x, t)>0, \quad 0 \leq a_{2} \bar{u}_{1}^{b_{2}}(x, t)-a_{2} u_{1}^{b_{2}}(x, t)<\frac{1}{2} \varepsilon_{1}(x, t),
$$

for $\left|x-x_{0}\right|<\eta, t_{0}<t<t_{0}+\delta(x)$. Therefore,

$$
\begin{aligned}
a_{2} \bar{u}_{1}^{b_{2}}(x, t) & <a_{2} u_{1}^{b_{2}}(x, t)+\frac{1}{2} \varepsilon_{1}(x, t) \\
& =a_{2} u_{1}^{b_{2}}(x, t)+\frac{1}{2} \bar{u}_{2}(x, t)-\frac{1}{2} a_{2} u_{1}^{b_{2}}(x, t) \\
& =-\frac{1}{2} a_{2} u_{1}^{b_{2}}(x, t)+\frac{1}{2} \bar{u}_{2}(x, t)<\bar{u}_{2}(x, t)
\end{aligned}
$$

for $\left|x-x_{0}\right|<\eta, t_{0}<t<t_{0}+\delta(x)$.

By assumption, $\bar{u}\left(x, t_{0}\right) \in \partial M$ so it follows that locally $\bar{u}(x, t) \in M$, and in particular

$$
\bar{u}_{2}(x, t) \leq a_{2} \bar{u}_{1}^{b_{2}}(x, t), \quad x \in \mathbb{R}^{N}, t_{0}<t<t_{0}+\delta^{\prime}(x) .
$$

Thus, for $\left|x-x_{0}\right|<\eta, t_{0}<t<t_{0}+\min \left\{\delta(x), \delta^{\prime}(x)\right\}$ we have a contradiction with (2.9). Finally, we infer that

$$
F(u(x, t)) \leq \Theta \quad \text { for } x \in \mathbb{R}^{N}, t \in[0, T),
$$

i.e. $M$ is globally invariant.

REMARK 2.7. We can always make the numbers $\alpha_{i}$ into a decreasing sequence. Namely, we find a permutation $\sigma$ such that $\sigma^{-1}(i+1) \leq \sigma^{-1}(i)$, $i=1, \ldots, m-1$. Then, putting $\sigma^{-1}(i)$ instead of $i$ in $u_{i}, i=1, \ldots, m$, and consequently in $\alpha_{i}, d_{i}, p_{k}^{i}$, we get $\alpha_{j+1} \leq \alpha_{j}$. Thus, the assumption $b_{j+1} \leq b_{j}$ is natural.

3. Case $p_{1}^{1}>1$. In this part we consider separately the case $p_{1}^{1}>1$ proving Theorem 1. The method used to obtain blow up results in this case is based on some lower bounds and does not require applying an invariant region.

In further considerations we use the notion of a subsolution and a supersolution of the system (1.1) and the comparison principles. The related 
definitions and theorems can be found in [EL] (Lemmas A1, A2 in Appendices) and [LS] (Definition 1.1, Theorem 1.2).

First, our goal is to show the existence of global solutions of (1.1) under the assumptions of part A of Theorem 1. To establish this assertion, we look for a global supersolution of the form

$$
\bar{u}_{i}=\varepsilon_{i}\left(t+t_{0}\right)^{\beta_{i}-N / 2} \exp \left(\frac{-|x|^{2}}{4 d_{i}\left(t+t_{0}\right)}\right)
$$

with some positive constants $\varepsilon_{i}, \beta_{i}, i=1, \ldots, m$.

Consider the system

$$
\bar{u}_{i t}-d_{i} \Delta \bar{u}_{i} \geq \prod_{k=1}^{m} \bar{u}_{k}^{p_{k}^{i}}
$$

Substituting (3.1) to (3.2) we obtain

$$
\frac{N}{2}\left(\sum_{k=1}^{m} p_{k}^{i}-1\right)-1>\sum_{k=1}^{m} p_{k}^{i} \beta_{i}-\beta_{i}, \quad i=1, \ldots, m,
$$

provided that $\varepsilon_{i}$ are sufficiently small and $t_{0}$ is large enough. By assumption $\sum_{k=1}^{m} p_{k}^{i} \geq \sum_{k=1}^{m} p_{k}^{1}>2 / N+1$, so the left-hand sides of (3.3) are positive and we can find small positive $\beta_{i}, i=1, \ldots, m$, which satisfy (3.3). Thus, the functions $\bar{u}_{i}, i=1, \ldots, m$, are supersolutions of (1.1), so the system has global solutions.

Now we prove the blow up results. We shall derive some lower bounds for solutions of (1.1) which eventually lead to establishing the assertions of Theorem 1. For simplicity, we denote here $p_{1}^{1}$ by $p$.

LEMMA 3.1. Suppose that $p=p_{1}^{1}>1$. Let $t_{1} \in(0, T)$ and for all $x \in \mathbb{R}^{N}$, $v(x)=\min _{i} u_{i}\left(x, t_{1}\right)>0$. Then

$$
u_{1}(x, t) \geq \sum_{n=0}^{j}(\theta t)^{\left(p^{n}-1\right) /(p-1)}\left(d^{N / 2} S_{\min }(t) v(x)\right)^{\pi_{n}}
$$

for $j=1,2, \ldots, x \in \mathbb{R}^{N}, t \in\left(0, T-2 t_{1}\right)$ where $\theta, d>0$ are some constants, $S_{\min }$ is the semigroup operator for the heat equation with diffusion coefficient $\min _{k} d_{k}$, and

$$
\pi_{n}=p^{n}+\sum_{k=2}^{m} p_{k}^{1} \frac{p^{n}-1}{p-1} .
$$

P r o of. Using a rescaling argument in formulas (2.2) we observe that

$$
u_{i}(t) \geq S_{i}(t) v \geq d^{N / 2} S_{\min }(t) v
$$

where $d=\min _{k} d_{k} / \max _{k} d_{k}$ and $t \in\left(0, T-2 t_{1}\right)$. To obtain the estimate we 
apply (3.5) in (2.2) for $i=1$ and the Jensen inequality for $\sum_{k=1}^{m} p_{k}^{1} \geq p>1$ to get

(3.6) $\quad u_{1}(t) \geq d^{N / 2} S_{\min }(t) v$

$$
\begin{gathered}
+d^{(N / 2)\left(1+\sum_{k=1}^{m} p_{k}^{1}\right)} \int_{0}^{t} S_{\min }(t-s)\left(S_{\min }(s) v\right)^{\sum_{k=1}^{m} p_{k}^{1}} d s \\
\geq d^{N / 2} S_{\min }(t) v+d^{(N / 2)\left(1+\sum_{k=1}^{m} p_{k}^{1}\right)} t\left(S_{\min }(t) v\right)^{\sum_{k=1}^{m} p_{k}^{1}} .
\end{gathered}
$$

In this way, we have established our lemma for $j=1$. Further, we assume that

$$
u_{1}(t) \geq \sum_{n=0}^{j} C_{n} d_{n} t^{\gamma_{n}}\left(S_{\min }(t) v\right)^{\pi_{n}}
$$

where

$$
\begin{aligned}
& \gamma_{n+1}=\gamma_{n} p+1, \quad \pi_{n+1}=\pi_{n} p+\sum_{k=2}^{m} p_{k}^{1}, \\
& C_{n+1}=C_{n}^{p} / \gamma_{n+1}, \quad d_{n+1}=d_{n}^{p} d^{(N / 2)\left(\sum_{k=2}^{m} p_{k}^{1}+1\right)},
\end{aligned}
$$

We see that $\pi_{0}=1$, and since $p>1$ we find that $\pi_{n}>1$ for all $n$ and we can use Jensen's inequality. By applying the inequality $(a+b)^{p} \geq a^{p}+b^{p}$ for $a, b>0, p \geq 1$, we deduce from $(2.2),(3.5),(3.7)$ that

$$
\begin{aligned}
u_{1}(t)- & d^{N / 2} S_{\min }(t) v \\
\geq & \int_{0}^{t} S_{1}(t-s) \prod_{k=2}^{m}\left(S_{k}(s) v\right)^{p_{k}^{1}}\left(\sum_{n=0}^{j} C_{n} s^{\gamma_{n}} d_{n}\left(S_{\min }(s) v\right)^{\pi_{n}}\right)^{p} d s \\
\geq & d^{(N / 2)\left(1+\sum_{k=2}^{m} p_{k}^{1}\right)} \int_{0}^{t} S_{\min }(t-s)\left(S_{\min }(s) v\right)^{\sum_{k=2}^{m} p_{k}^{1}} \\
& \quad \times \sum_{n=0}^{j} d_{n}^{p} C_{n}^{p} s^{p \gamma_{n}}\left(S_{\min }(s) v\right)^{p \pi_{n}} d s \\
\geq & d^{(N / 2)\left(1+\sum_{k=2}^{m} p_{k}^{1}\right)} \sum_{n=0}^{j} \frac{C_{n}^{p}}{p \gamma_{n}+1} t^{p \gamma_{n}+1} d_{n}^{p}\left(S_{\min }(t) v\right)^{p \pi_{n}+\sum_{k=2}^{m} p_{k}^{1}}
\end{aligned}
$$

i.e.

$$
u_{1}(t) \geq \sum_{n=0}^{j+1} C_{n} d_{n} t^{\gamma_{n}}\left(S_{\min }(t) v\right)^{\pi_{n}}
$$

Notice that (3.9) is (3.7) for $j+1$ and by (3.8) we can compute that

$$
\begin{aligned}
\gamma_{n} & =\frac{p^{n}-1}{p-1}, \quad \pi_{n}=p^{n}+\sum_{k=2}^{m} p_{k}^{1} \frac{p^{n}-1}{p-1}, \quad d_{n}=d^{N \pi_{n} / 2} \\
C_{n} & =\frac{C_{n-1}^{p}}{p^{n}-1}(p-1)=\frac{C_{n-2}^{p^{2}}(p-1)^{p+1}}{\left(p^{n}-1\right)\left(p^{n-1}-1\right)^{p}}=\prod_{l=1}^{n}\left(\frac{p-1}{p^{l}-1}\right)^{p^{n-l}} .
\end{aligned}
$$


To estimate $C_{n}$ from below we observe that

$$
\begin{aligned}
\ln C_{n} & \geq \sum_{l=1}^{n} p^{n-l}\left[\ln (p-1)-\ln \left(p^{l}-1\right)\right] \\
& \geq \frac{p^{n}-1}{p-1} \ln (p-1)-\sum_{l=1}^{n} l p^{n-l} \ln p \\
& \geq \frac{p^{n}-1}{p-1} \ln (p-1)-p^{n-1} \ln p \sum_{l=1}^{\infty} l p^{-l+1} \\
& \geq \frac{p^{n}-1}{p-1}\left(\ln (p-1)-\frac{p^{2}}{(p-1)^{2}} \ln p\right) \\
& =\frac{p^{n}-1}{p-1} \ln \left((p-1) / p^{p^{2} /(p-1)^{2}}\right) .
\end{aligned}
$$

Therefore we have found $\theta=(p-1) p^{-p^{2} /(p-1)^{2}}>0$ such that $C_{n} \geq \theta^{\gamma_{n}}$. Thus, by (3.7) and (3.10), the proof is complete.

Proof of Theorem 1A. The existence of nonglobal solutions in the case $p_{1}^{1}>1$ (Theorem $1 \mathrm{~A}$ ) is now a consequence of Lemma 3.1. Lemma 2.1 implies that $\min _{i} u_{i}(x, t) \geq C e^{-A|x|^{2}}$, so

$$
S_{\min }(t) v(x) \geq C\left(1+4 A_{0} t\right)^{-N / 2} \exp \left(\frac{-A|x|^{2}}{1+4 A_{0} t}\right)
$$

where $A_{0}=A\left(\min _{i} d_{i}\right)$.

Now put $x=0$ and fix $t_{0}$ such that $\theta t_{0}>1$ (i.e. $t_{0}>p^{p^{2} /(p-1)^{2}} /(p-1)$ by (3.11)). We remark that by the assumption on the initial data we can make $C$ as large as we wish. In particular, we can take $C>2\left[\left(1+4 A_{0} t_{0}\right) / d\right]^{N / 2}$. Then, by (3.4),

$$
u_{1}\left(0, t_{0}\right)>2(j+1) \quad \text { for } j=1,2, \ldots
$$

and this contradicts the boundedness of $u_{1}$. Thus, $u$ is nonglobal.

LEMMA 3.2. Suppose that $p_{1}^{1}=p>1$ and $\left(\sum_{k=1}^{m} p_{k}^{1}-1\right) N / 2 \leq 1$. If $u=\left(u_{1}, \ldots, u_{m}\right)$ is a nondegenerate solution of (1.1) satisfying, for some $C_{0}$ and $A>0$,

$$
\min _{i} u_{i}(0) \geq C_{0} \exp \left(-A|x|^{2}\right)
$$

then there exist positive $C, \theta>0$ such that

$$
u_{1}(x, t)>C(1+4 A t)^{-N / 2} \sum_{n=0}^{j}\left[\theta \ln \left(1+4 A d_{0} t\right)^{\gamma_{n}} \exp \left(\frac{-A \pi_{n}|x|^{2}}{1+4 A d_{0} t}\right)\right]
$$

where $d_{0}=\min \left(d_{1}, \ldots, d_{m}, 1\right), j=1,2, \ldots$ and $\gamma_{n}, \pi_{n}$ are given by $(3.10)$. 
Proof. By Lemma 2.1, the assumption (3.14) is satisfied. Then using formulas (2.2) we obtain

$$
\begin{aligned}
\min _{i} u_{i}(x, t) & \geq \min _{i}\left(S_{i}(t) u_{0 i}\right) \geq d^{N / 2} \min _{i}\left(S_{0}(t) u_{0 i}\right) \\
& \geq d^{N / 2} C_{0}\left(1+4 A d_{0} t\right)^{-N / 2} \exp \left(\frac{-A|x|^{2}}{1+4 A d_{0} t}\right)
\end{aligned}
$$

where $S_{0}$ is the semigroup operator for the heat equation with diffusion coefficient $d_{0}$ and $d=d_{0} / \max _{k} d_{k}$.

The proof is very similar to that of Lemma 3.1. Using (3.16) in (2.2) we want to get (3.15) for $j=1$. We have

$$
\begin{aligned}
& u_{1}(x, t) \geq d^{N / 2} C_{0}\left(1+4 A d_{0} t\right)^{-N / 2} \exp \left(\frac{-A|x|^{2}}{1+4 A d_{0} t}\right) \\
& +d^{(N / 2)\left(\sum_{k=1}^{m} p_{k}^{1}+1\right)} C_{0}^{\sum_{k=1}^{m} p_{k}^{1}} \int_{0}^{t}\left(1+4 A d_{0} s\right)^{-(N / 2) \sum_{k=1}^{m} p_{k}^{1}} \\
& \times S_{0}(t-s) \exp \left(\frac{-A \sum_{k=1}^{m} p_{k}^{1}|x|^{2}}{1+4 A d_{0} s}\right) d s \\
& =d^{N / 2} C_{0}\left(1+4 A d_{0} t\right)^{-N / 2} \exp \left(\frac{-A|x|^{2}}{1+4 A d_{0} t}\right) \\
& +d^{N / 2}\left(d^{N / 2} C_{0}\right)^{\sum_{k=1}^{m} p_{k}^{1}} \int_{0}^{t}\left(1+4 A d_{0} s\right)^{-(N / 2)\left(\sum_{k=1}^{m} p_{k}^{1}-1\right)} \\
& \times\left[1+4 A d_{0} s+4 A \sum_{k=1}^{m} p_{k}^{1}(t-s)\right]^{-N / 2} \\
& \times \exp \left(\frac{-A \sum_{k=1}^{m} p_{k}^{1}|x|^{2}}{1+4 A d_{0} s+4 A \sum_{k=1}^{m} p_{k}^{1}(t-s)}\right) d s .
\end{aligned}
$$

Putting

$$
f(s)=1+4 A d_{0} s+4 A \sum_{k=1}^{m} p_{k}^{1}(t-s)
$$

we notice that $f^{\prime}(s)=4 A\left(d_{0}-\sum_{k=1}^{m} p_{k}^{1}\right)<0$ as $\sum_{k=1}^{m} p_{k}^{1} \geq p>1 \geq d_{0}$. This implies

$$
\begin{aligned}
u_{1}(x, t) \geq & d^{N / 2} C_{0}\left(1+4 A d_{0} t\right)^{-N / 2} \exp \left(\frac{-A|x|^{2}}{1+4 A d_{0} t}\right) \\
& +d^{N / 2}\left(d^{N / 2} C_{0}\right)^{\sum_{k=1}^{m} p_{k}^{1}}\left(1+4 A \sum_{k=1}^{m} p_{k}^{1} t\right)^{-N / 2} \\
& \times \exp \left(\frac{-A \sum_{k=1}^{m} p_{k}^{1}|x|^{2}}{1+4 A d_{0} t}\right) \int_{0}^{t}\left(1+4 A d_{0} s\right)^{-(N / 2)\left(\sum_{k=1}^{m} p_{k}^{1}-1\right)} d s .
\end{aligned}
$$


Finally, using $\left(\sum_{k=1}^{m} p_{k}^{1}-1\right) N / 2 \leq 1$ we obtain

$$
\begin{aligned}
u_{1}(x, t) \geq & C_{0}\left(d d_{0}\right)^{N / 2}\left(1+4 A d_{0} t\right)^{-N / 2} \exp \left(\frac{-A|x|^{2}}{1+4 A d_{0} t}\right) \\
& +\frac{\left(d d_{0}\right)^{N / 2}\left(d^{N / 2} C_{0}\right)^{\sum_{k=1}^{m} p_{k}^{1}}}{\left(\sum_{k=1}^{m} p_{k}^{1}\right)^{N / 2}}\left(1+4 A d_{0} t\right)^{-N / 2} \\
& \times \exp \left(\frac{-A \sum_{k=1}^{m} p_{k}^{1}|x|^{2}}{1+4 A d_{0} t}\right) \ln \left(1+4 A d_{0} t\right),
\end{aligned}
$$

which is our statement for $j=1$. Arguing by induction, we assume that (3.18) $u_{1}(x, t)$

$$
\geq\left(1+4 A d_{0} t\right)^{-N / 2} \sum_{n=0}^{j} C_{n} \exp \left(\frac{-A \pi_{n}|x|^{2}}{1+4 A d_{0} t}\right)\left[\ln \left(1+4 A d_{0} t\right)\right]^{\gamma_{n}}
$$

where

$$
\begin{aligned}
C_{n+1} & =\frac{\left(C_{0} d^{N / 2}\right)^{\sum_{k=2}^{m} p_{k}^{1}}\left(d d_{0}\right)^{N / 2} C_{n}^{p}}{\left(p \pi_{n}+\sum_{k=2}^{m} p_{k}^{1}\right)^{N / 2}\left(\gamma_{n} p+1\right)} \\
\gamma_{n+1} & =p \gamma_{n}+1, \quad \gamma_{0}=0 \\
\pi_{n+1} & =p \pi_{n}+\sum_{k=2}^{m} p_{k}^{1}, \quad \pi_{0}=1 .
\end{aligned}
$$

We employ (3.18) and (3.16) in (2.2). Then

$$
\begin{aligned}
u_{1}(x, t) & \\
\geq & C_{0} d^{N / 2}(1+4 A t)^{-N / 2} \exp \left(\frac{-A|x|^{2}}{1+4 A d_{0} t}\right)+d^{(N / 2)\left(\sum_{k=2}^{m} p_{k}^{1}+1\right)} C_{0}^{\sum_{k=2}^{m} p_{k}^{1}} \\
& \times \int_{0}^{t}\left(1+4 A d_{0} s\right)^{-(N / 2) \sum_{k=1}^{m} p_{k}^{1}} \exp \left(\frac{-A \sum_{k=2}^{m} p_{k}^{1}|x|^{2}}{1+4 A d_{0} s}\right) \\
& \times S_{0}(t-s)\left[\sum_{n=1}^{j} C_{n} \exp \left(\frac{-A \pi_{n}|x|^{2}}{1+4 A d_{0} s}\right)\left(\ln \left(1+4 A d_{0} s\right)\right)^{\gamma_{n}}\right]^{p} d s \\
\geq & C_{0} d^{N / 2}(1+4 A t)^{-N / 2} \exp \left(\frac{-A|x|^{2}}{1+4 A d_{0} t}\right) \\
& +d^{(N / 2)\left(\sum_{k=2}^{m} p_{k}^{1}+1\right)} C_{0}^{\sum_{k=2}^{m} p_{k}^{1}} \int_{0}^{t}\left(1+4 A d_{0} s\right)^{-(N / 2)} \sum_{k=1}^{m} p_{k}^{1} \\
& \times S_{0}(t-s) \sum_{n=1}^{j} C_{n}^{p} \exp \left(\frac{-A\left(p \pi_{n}+\sum_{k=2}^{m} p_{k}^{1}\right)|x|^{2}}{1+4 A d_{0} s}\right) \\
& \times\left(\ln \left(1+4 A d_{0} s\right)\right)^{p \gamma_{n}} d s
\end{aligned}
$$




$$
\begin{aligned}
\geq & C_{0} d^{N / 2}(1+4 A t)^{-N / 2} \exp \left(\frac{-A|x|^{2}}{1+4 A d_{0} t}\right) \\
& +d^{N / 2}\left(C_{0} d^{N / 2}\right)^{\sum_{k=2}^{m} p_{k}^{1}} \int_{0}^{t}\left(1+4 A d_{0} s\right)^{-(N / 2)\left(\sum_{k=1}^{m} p_{k}^{1}-1\right)} \\
& \times \sum_{n=1}^{j} C_{n}^{p}\left(1+4 A d_{0} s+4 A \pi_{n+1}(t-s)\right)^{-N / 2}\left(\ln \left(1+4 A d_{0} s\right)\right)^{p \gamma_{n}} \\
& \times \exp \left(\frac{-A\left(p \pi_{n}+\sum_{k=2}^{m} p_{k}^{1}\right)|x|^{2}}{1+4 A d_{0} s+4 A\left(p \pi_{n}+\sum_{k=2}^{m} p_{k}^{1}\right)(t-s)}\right) d s
\end{aligned}
$$

As in the case $j=1$ we observe that

$$
f_{k}(s)=1+4 A d_{0} s+4 A\left(p \pi_{n}+\sum_{k=2}^{m} p_{k}^{1}\right)(t-s)
$$

satisfies

$$
f_{k}^{\prime}(s)=4 A\left(d_{0}-\left(p \pi_{n}+\sum_{k=2}^{m} p_{k}^{1}\right)\right) s<0
$$

because $\pi_{n+1} \geq \pi_{n} \geq p>d_{0}$ for $n=1, \ldots, m$ by (3.19). Employing also $\left(\sum_{k=1}^{m} p_{k}^{1}-1\right) N / 2 \leq 1$ we obtain

$$
\begin{aligned}
& u_{1}(x, t) \\
& \geq C_{0} d^{N / 2}(1+4 A t)^{-N / 2} \exp \left(\frac{-A|x|^{2}}{1+4 A d_{0} t}\right)+d^{N / 2}\left(C_{0} d^{N / 2}\right)^{\sum_{k=2}^{m} p_{k}^{1}} \\
& \quad \times \sum_{n=1}^{j}\left(C_{n}\right)^{p}\left(1+4 A \pi_{n+1} t\right)^{-N / 2} \exp \left(\frac{-A \pi_{n+1}|x|^{2}}{1+4 A d_{0} t}\right) \\
& \quad \times \int_{0}^{t} \frac{\left[\ln \left(1+4 A d_{0} s\right)\right]^{p \gamma_{n}}}{1+4 A d_{0} s} d s \\
& \geq C_{0}\left(d d_{0}\right)^{N / 2}\left(1+4 A d_{0} t\right)^{-N / 2} \exp \left(\frac{-A|x|^{2}}{1+4 A d_{0} t}\right) \\
& \quad+\left(1+4 A d_{0} t\right)^{-N / 2} d^{N / 2}\left(C_{0} d^{N / 2}\right)^{\sum_{k=2}^{m} p_{k}^{1}} \\
& \quad \times \sum_{n=1}^{j} \frac{\left(C_{n}\right)^{p} d_{0}^{N / 2}}{\pi_{n+1}^{N / 2}\left(p \gamma_{n}+1\right)} \exp \left(\frac{-A \pi_{n+1}|x|^{2}}{1+4 A d_{0} t}\right)\left[\ln \left(1+4 A d_{0} t\right)\right]^{p \gamma_{n+1}} \\
& \geq \\
& \quad\left(1+4 A d_{0} t\right)^{-N / 2} \sum_{n=0}^{j+1} C_{n} \exp \left(\frac{-A \pi_{n}|x|^{2}}{1+4 A d_{0} t}\right)\left[\ln \left(1+4 A d_{0} t\right)\right]^{\gamma_{n}},
\end{aligned}
$$

so we have proved that (3.18) holds with $j+1$ in place of $j$. It remains to estimate $C_{n}$, because by $(3.19), \pi_{n}$ and $\gamma_{n}$ are as in the preceding lemma, 
i.e.

$$
\gamma_{n}=\frac{p^{n}-1}{p-1}, \quad \pi_{n}=p^{n}+\sum_{k=2}^{m} p_{k}^{1} \frac{p^{n}-1}{p-1}
$$

We compute

$$
\begin{aligned}
\ln C_{n+1}= & p \ln C_{n}+\frac{N}{2} \ln d_{0}+\frac{N}{2}\left(\sum_{k=2}^{m} p_{k}^{1}+1\right) \ln d \\
& +\sum_{k=2}^{m} p_{k}^{1} \ln C_{0}-\frac{N}{2} \ln \pi_{n}-\ln \gamma_{n} \\
\geq & p \ln C_{n}-\frac{N}{2} \ln \left[\left(1+\sum_{k=2}^{m} p_{k}^{1} \frac{1}{p-1}\right) p^{n}\right]-\ln \frac{p^{n}}{p-1} \\
& +\frac{N}{2}\left(\ln d_{0}+\left(\sum_{k=2}^{m} p_{k}^{1}+1\right) \ln d\right)+\sum_{k=2}^{m} p_{k}^{1} \ln C_{0} \\
\geq & p \ln C_{n}-\left(\frac{N}{2}+1\right) n \ln p-a
\end{aligned}
$$

where $a$ does not depend on $n$. This implies

$$
\begin{aligned}
\ln C_{n+1} & \geq p^{n} \ln C_{0}+\frac{a\left(p^{n+1}-1\right)}{p-1}-\ln p\left(\frac{N}{2}+1\right) \sum_{k=0}^{n}(n-k) p^{k} \\
& \geq p^{n+1}\left[\frac{\ln C_{0}}{p}-\frac{a\left(1-p^{-n-1}\right)}{p-1}-\ln p\left(\frac{N}{2}+1\right) \sum_{k=0}^{\infty} k p^{-k-1}\right] \\
& \geq p^{n+1}\left[\frac{\ln C_{0}}{p}-\frac{|a|}{p-1}-\ln p\left(\frac{N}{2}+1\right) \frac{p^{2}}{(p-1)^{2}}\right] .
\end{aligned}
$$

By (3.22) we can find $D>0$ such that

$$
\ln C_{n} \geq-p^{n} D \geq-D p \frac{p^{n}-1}{p-1}=\ln \theta^{\gamma_{n}}
$$

where $\theta=e^{-D p}>0$. Thus $C_{n} \geq \theta^{\gamma_{n}}$ and therefore, by (3.20) and (3.21) the proof is complete.

Proof of Theorem 1B. Let us take into account (3.15). For $x=0$ and $t_{0}$ such that $\theta \ln \left(1+4 A d_{0} t_{0}\right) \geq 1$ we obtain

$$
u_{1}\left(0, t_{0}\right) \geq C\left(1+4 A t_{0}\right)^{-N / 2}(j+1) \quad \text { for } j=1,2, \ldots,
$$

which implies that for $t \geq t_{0}$ the solution of (1.1) is not bounded. This establishes Theorem 1B.

4. Case $p_{1}^{1}<1$. We prove Theorem 2 using comparison principles (see Lemmas A1, A2 in [EL], Theorem 1.2 in [LS]). Applying a minimal subsolu - 
tion or maximal supersolution instead of a subsolution or supersolution, we can omit the Lipschitz continuity requirement for the nonlinear functions in the system. This makes it possible to establish blow up or global existence of solutions.

Proof of Theorem 2A. Consider a solution $u^{*}(t)$ of the kinetic system (2.3) with initial value $u_{0}^{*}$ defined as follows:

$$
u_{0 i}^{*}=\sup _{\mathbb{R}^{N}} u_{0 i}(x) \geq u_{0 i}(x), \quad i=1, \ldots, m .
$$

Then $u^{*}(t)$ is also a space-independent solution of (1.1) and by the comparison theorem

$$
0 \leq u(x, t) \leq u^{*}(t)
$$

As $0<r<1$ we apply Lemma 2.4(i) to the solution $u^{*}(t)$ of $(2.3)$. Therefore, (4.1) implies that $u(x, t)$ exists globally since $u^{*}(t)$ does.

Proof of Theorem 2B. First, we take $u_{0}(x) \in \partial M$, so by Lemma 2.6, $u(x, t) \in M$, i.e. $0 \leq u_{i+1}(x, t) \leq a_{i+1}\left(u_{i}(x, t)\right)^{b_{i+1} / b_{i}}$ for $(x, t) \in \mathbb{R}^{N} \times[0, T)$. Applying this inequality to the system (1.1), we get, by (2.5),

$$
u_{i t}-d_{i} \Delta u_{i} \leq\left(\prod_{k=1}^{m} \prod_{j=1}^{k} a_{j}^{1 / b_{j}} u_{1}\right)^{\left(1+\alpha_{i}\right) / \alpha_{1}}, \quad i=1, \ldots, m .
$$

Thus, (1.1) can be compared with the following supersystem:

$$
\left\{\begin{array}{l}
\bar{u}_{i t}-d_{i} \Delta \bar{u}_{i}=A_{i} \bar{u}_{1}^{\left(1+\alpha_{i}\right) / \alpha_{1}} \\
\bar{u}_{i}(x, 0)=u_{0 i}(x), \quad i=1, \ldots, m
\end{array}\right.
$$

where $A_{i}=\left(\prod_{k=1}^{m} \prod_{j=1}^{k} a_{j}^{1 / b_{j}}\right)^{\left(1+\alpha_{i}\right) / \alpha_{1}}$, and then

$$
\bar{u}(x, t) \geq u(x, t) .
$$

We note that $\left(1+\alpha_{1}\right) / \alpha_{1}=r>1+2 / N$. We apply the Fujita theorem to the first equation of (4.2). Thus, for $u_{01}(x)$ sufficiently small, $\bar{u}_{1}(x, t)$ exists globally. Using $(4.2)_{i}$ we infer that $\bar{u}_{i}(x, t)$ also exists globally for $i=2, \ldots, m$ and hence so does $u(x, t)=\left(u_{1}(x, t), \ldots, u_{m}(x, t)\right)$ by $(4.3)$.

Next, we take $u_{0}(x) \notin \partial M$ and choose $u_{01}^{*}(x)$ such that

$$
0 \leq u_{0 i} \leq \prod_{j=1}^{i} a_{j}^{b_{i} / b_{j}}\left(u_{01}^{*}\right)^{b_{i}}=u_{0 i}^{*} .
$$

Then a solution $u^{*}(x, t)$ of $(1.1)$ has its initial values $u_{0}^{*}(x)$ on $\partial M$ and we can apply the above considerations. Moreover, by comparison,

$$
0 \leq u(x, t) \leq u^{*}(x, t),
$$

so by the global existence of $u^{*}(x, t)$ we get the assertion for $u(x, t)$. 
Next, we argue similarly. If $u_{0}(x) \in \partial M$, then by Lemma 2.6, $u(x, t) \in$ $M$, and (1.1) takes the form

$$
u_{i t}-d_{i} \Delta u_{i} \geq\left(\prod_{k=1}^{m} \prod_{j=k+1}^{m} a_{j}^{-1 / b_{j}} u_{m}^{1 / b_{m}}\right)^{\left(1+\alpha_{i}\right) / \alpha_{1}}
$$

because $u_{k} \geq\left(\prod_{j=k+1}^{m} a_{j}^{-1 / b_{j}} u_{m}^{1 / b_{m}}\right)^{b_{k}}$.

We consider a subsystem corresponding to (1.1):

$$
\left\{\begin{array}{l}
\underline{u}_{i t}-d_{i} \Delta \underline{u}_{i}=B_{i} \underline{u}_{m}^{\left(1+\alpha_{i}\right) / \alpha_{m}}, \\
\underline{u}_{i}(x, 0)=u_{0 i}(x), \quad i=1, \ldots, m,
\end{array}\right.
$$

where $B_{i}=\left(\prod_{k=1}^{m} \prod_{j=k+1}^{m} a_{j}^{-1 / b_{j}}\right)^{\left(1+\alpha_{i}\right) / \alpha_{1}}$. Using the comparison principle to systems (1.1) and (4.4) we have

$$
\underline{u}(x, t) \leq u(x, t) .
$$

By assumption

$$
\frac{1+\alpha_{m}}{\alpha_{m}}=1+\frac{1}{\alpha_{m}} \geq 1+\frac{1}{\alpha_{1}}=r>1+\frac{2}{N}
$$

so we can apply the Fujita theorem to the last equation of (4.4). Hence, $\underline{u}_{m}(x, t)$ blows up in finite time provided $\underline{u}_{m}(x, 0)=u_{0 m}(x)$ is large enough. By $(4.4)_{i}, i=1, \ldots, m-1$, we see that $\underline{u}_{i}(x, t)$ does not exist globally; by $(4.5)$ also $u(x, t)=\left(u_{1}(x, t), \ldots, u_{m}(x, t)\right)$ blows up in finite time.

If $u_{0}(x) \notin \partial M$, by Lemma 2.1 we can choose $u_{01}^{*}(x)$ satisfying

$$
0 \leq u_{0 i}^{*}=\prod_{j=1}^{i} a_{j}^{b_{i} / b_{j}}\left(u_{01}^{*}\right)^{b_{i}} \leq u_{0 i} .
$$

Next, we complete the proof using the same argument as in the proof of the previous part.

The proof of Theorem $2 \mathrm{C}$ is based on a construction of subsolutions to a system of reaction-diffusion equations (see [LS]). Adapting this result to a system of $m$ equations, we get the following lemma.

LEMMA 4.1. Let

$$
\left\{\begin{array}{l}
u_{i t}=d_{i} \Delta u_{i}+f_{i}\left(u_{1}, \ldots, u_{m}\right), \\
u_{i}(x, 0)=u_{0 i}(x) \geq 0, \quad i=1, \ldots, m .
\end{array}\right.
$$

Let $u_{i}=\phi_{i}\left(t, u_{01}, \ldots, u_{m_{0}}\right), i=1, \ldots, m$, be a solution of the kinetic system

$$
\left\{\begin{array}{l}
u_{i t}=f_{i}\left(u_{1}, \ldots, u_{m}\right), \\
u_{i}(0)=u_{0 i}, \quad i=1, \ldots, m .
\end{array}\right.
$$

Putting $z(x, t)=S_{m}(t) v^{*}$ (then $\left.z_{t}=d_{m} \Delta z, z(0)=v^{*}(x)\right)$ we define

$$
\Phi_{i}(x, t)=\phi_{i}(t, 0, \ldots, 0, z(x, t)), \quad i=1, \ldots, m .
$$


Then $\Phi(x, t)=\left(\Phi_{1}(x, t), \ldots, \Phi_{m}(x, t)\right)$ is a subsolution to (4.6) iff $\Phi_{1}(0)=$ $v^{*}(x) \leq u_{0 m}(x)$ and $\phi_{i v v} \geq 0, i=1, \ldots, m$.

Proof of Theorem 2C. Let $f_{i}\left(u_{1}, \ldots, u_{m}\right)=\prod_{k=1}^{m} u_{k}^{p_{k}^{i}}$. Then (4.6) takes the form of (1.1). Assume that $\min _{k} \alpha_{k}=\alpha_{m}$, i.e. $b_{k} \geq b_{m}, i=1, \ldots, m$. Setting the initial values in the kinetic system (4.7) as follows: $u_{m}(0)=v$, $u_{i}(0)=\prod_{j=i+1}^{m} a_{j}^{-b_{i} / b_{j}} v^{b_{i} / b_{m}}, i=1, \ldots, m-1$, we obtain the following solution of this system:

$$
\phi_{i}(t, v)=c_{i}\left[v^{1-q}-A_{*}(q-1) t\right]^{-b_{i} /\left(b_{m}(q-1)\right)},
$$

where $c_{m}=1, c_{i}=\prod_{j=i+1}^{m} a_{j}^{-b_{i} / b_{j}}, A_{*}=b_{m} c_{1}^{1 / \alpha_{1}}$ and $q=1+1 / \alpha_{m}$. Thus

$$
\phi_{i}(t)=c_{i} \phi_{m}^{b_{i} / b_{m}}(t) .
$$

We assume that $0 \leq v^{*}(x) \leq u_{0 m}(x), c_{i}\left(v^{*}(x)\right)^{b_{i} / b_{m}} \leq u_{0 i}(x)$, where $u_{0}(x)$ is the initial value in (1.1). Let $\Phi(t, z(x, t))$ be given by $(4.8)$ and (4.9). Then, by Lemma 4.1, if $\phi_{i v v} \geq 0, i=1, \ldots, m$, then $\Phi(t, z(x, t))$ is a subsolution to (1.1).

By (4.9) we get

$$
\phi_{m v v}=\phi_{m}^{q} q v^{-1-q}\left[\left(\frac{\phi_{m}}{v}\right)^{q-1}-1\right],
$$

and so, since $\phi_{m}^{1-q}<v^{1-q}, \phi_{m v v} \geq 0$ for $t \geq 0$. Using (4.10), we also have, for $i=1, \ldots, m-1$,

$$
\phi_{i v v}=c_{i} \frac{b_{i}}{b_{m}} \phi_{m}^{b_{i} / b_{m}-1+q} v^{-1-q}\left[\left(\frac{b_{i}}{b_{m}}+q-1\right)\left(\frac{\phi_{m}}{v}\right)^{q-1}-q\right]
$$

so $\phi_{i v v} \geq 0$ for $i=1, \ldots, m-1$ iff

$$
A_{*}(q-1) t \geq \frac{b_{m}-b_{i}}{b_{m} q} v^{1-q} .
$$

This inequality holds for $t \geq 0$ since $q>1$ and $b_{i} \geq b_{m}, i=1, \ldots, m$.

Next, we show that the subsolution $\Phi(x, t)$ to $(1.1)$ blows up in finite time. Putting $x=0$ in $\Phi_{m}(x, t)$ we have

$$
\Phi_{m}(0, t)=\left[z^{1-q}(0, t)-A_{*}(q-1) t\right]^{-1 /(q-1)}=f(t)^{-1 /(q-1)}
$$

so $f(0)=z^{1-q}(0,0)=v^{*}(0)>0$.

Since $1<q<1+2 / N$, there exists $t^{*}>0$ such that $f\left(t^{*}\right)=0$, namely

$$
t^{*}=\left[A_{*}(q-1)\left(\int_{\mathbb{R}^{N}}\left(4 d_{1} \pi\right)^{-N / 2} e^{-|\xi|^{2} /\left(4 d_{1} t^{*}\right)} v^{*}(\xi) d \xi\right)^{q-1}\right]^{1 /(N(q-1) / 2-1)} .
$$

Therefore, for some $t^{* *} \in\left(0, t^{*}\right], \lim _{t \rightarrow t^{* *}} \Phi_{m}(0, t)=+\infty$. Consequently, also $u(x, t)$ blows up in finite time. 
5. Growth rate estimates. This section establishes an upper and a lower bound on the growth rate near the blow up time $T$. To get both estimates, we use an idea of invariant regions to replace (1.1) by a corresponding sub- or supersystem. The utility of the concept lies in reducing our system to another one, involving a scalar equation. It is remarkable that such a significant modification of nonlinear terms yields the bounds which are suggested by the kinetic system.

First, we prove a lower estimate on the blow up rate.

Proof of Theorem 3. Retaining the notations of Section 2 we check whether the initial values $u_{0}$ belong to $\partial M$. If this assumption is satisfied, we will consider a corresponding subsolution of (1.1) starting from the same initial data. Otherwise, we have to choose $\underline{u}_{01}(x)$ such that

$$
0 \leq \underline{u}_{0 i}=\prod_{j=1}^{i} a_{j}^{b_{i} / b_{j}}\left(\underline{u}_{01}\right)^{b_{i}} \leq u_{0 i} .
$$

We consider $u^{*}(x, t)$ satisfying (1.1) with initial values $\underline{u}_{0}$. Since $\underline{u}_{0} \in \partial M$ by (5.1), Lemma 2.6 implies that $u^{*}(x, t) \in M$, so (1.1) leads to

$$
\left\{\begin{array}{l}
u_{i t}^{*}-d_{i} \Delta u_{i}^{*} \geq B_{i}\left(u_{m}^{*}\right)^{\left(1+\alpha_{i}\right) / \alpha_{m}}, \\
u_{i}^{*}(x, 0)=\underline{u}_{0 i}(x), \quad i=1, \ldots, m,
\end{array}\right.
$$

where $B_{i}=\left(\prod_{k=1}^{m} \prod_{j=k+1}^{m} a_{j}^{-1 / b_{j}}\right)^{\left(1+\alpha_{i}\right) / \alpha_{1}}$.

If we take into account a subsystem

$$
\left\{\begin{array}{l}
\underline{u}_{i t}-d_{i} \Delta \underline{u}_{i}=B_{i} u_{m}^{\left(1+\alpha_{i}\right) / \alpha_{m}}, \\
\underline{u}_{i}(x, 0)=\underline{u}_{0 i}(x),
\end{array}\right.
$$

then by comparison $\underline{u}_{i}(x, t)<u_{i}(x, t), i=1, \ldots, m$. Moreover, we remark that the last equation in (5.2) has the form

$$
\underline{u}_{m t}-d_{i} \underline{u}_{m}=B_{m} \underline{u}_{m}^{1+1 / \alpha_{m}} .
$$

For any compact set $\Omega \subset \mathbb{R}^{N}$ and $U_{m}(t)=\max _{x \in \Omega} \underline{u}_{m}(x, t)$ we prove

LEMMA 5.1. If $\underline{u}_{m}$ satisfies (5.3) then $U_{m}(t)$ is Lipschitz continuous and

$$
U_{m}^{\prime}(t) \leq U_{m}^{r_{m}} \quad \text { a.e., where } r_{m}=1+1 / \alpha_{m} .
$$

Proof. Suppose that $x_{i} \in \Omega$ is such that

$$
U_{m}\left(t_{i}\right)=u\left(x_{i}, t_{i}\right) \quad \text { for } i=1,2 .
$$

Putting $h=t_{2}-t_{1}>0$ we can estimate 


$$
\begin{aligned}
U_{m}\left(t_{2}\right)-U_{m}\left(t_{1}\right) & \geq u_{m}\left(x_{1}, t_{2}\right)-u_{m}\left(x_{1}, t_{1}\right) \\
& =h u_{m t}\left(x_{1}, t_{1}\right)+o(h), \\
U_{m}\left(t_{2}\right)-U_{m}\left(t_{1}\right) & \leq u_{m}\left(x_{2}, t_{2}\right)-u_{m}\left(x_{2}, t_{1}\right) \\
& =h u_{m t}\left(x_{2}, t_{2}\right)+o(h) .
\end{aligned}
$$

This yields the Lipschitz continuity. By definition of $U_{m}, \Delta u_{m}\left(x_{i}, t_{i}\right) \leq 0$, so (5.5) implies

$$
\begin{aligned}
\frac{U_{m}\left(t_{2}\right)-U_{m}\left(t_{1}\right)}{t_{2}-t_{1}} & \leq u_{m t}\left(x_{2}, t_{2}\right)+o(1) \\
& \leq\left(u_{m}\left(x_{2}, t_{2}\right)\right)^{r_{m}}+o(1)=U_{m}\left(t_{2}\right)^{r_{m}}+o(1)
\end{aligned}
$$

and the assertion follows.

Continuation of the proof of Theorem 3. We conclude that (5.4) takes the form

$$
\int_{U_{m}(t)}^{U_{m}(\tau)} \frac{d\left(U_{m}\right)}{U_{m}^{r_{m}}} \leq T-t
$$

provided that $\int_{0}^{\infty} s^{-r_{m}} d s<\infty$. By integration, we get

$$
U_{m} \geq C_{0}(T-t)^{-1 /\left(r_{m}-1\right)}=C_{0}(T-t)^{-\alpha_{m}}
$$

with $C_{0}=\alpha_{m}^{-1}$. Since $\underline{u}_{i}(x, t) \in M$ we also have

$$
\max _{x \in \Omega} \underline{u}_{i}(x, t) \geq\left(\prod_{j=i+1}^{m} a_{j}^{-1 / b_{j}} U_{m}^{1 / b_{m}}\right)^{b_{i}} \geq C_{i} U_{m}^{\alpha_{i} / \alpha_{m}} \geq C(T-t)^{-\alpha_{i}}
$$

Thus, because $\underline{u}(x, t)$ is a subsolution,

$$
\max _{x \in \Omega} u_{i}(x, t) \geq C(T-t)^{-\alpha_{i}}, \quad i=1, \ldots, m,
$$

which concludes the proof.

Next, we prove an upper bound.

Proof of Theorem 4. We will proceed similarly to the previous proof to obtain the assertion for some supersolution. Then by comparison the same bound from above remains true for the solution of (1.1).

We set $\bar{u}_{0 i}(x)$ in the following way: if $u_{0}(x) \in \partial M$ then $\bar{u}_{0 i}(x)=u_{0 i}(x)$, whereas for $u_{0}(x) \notin \partial M$ we find $\bar{u}_{01}(x)$ such that

$$
0 \leq u_{0 i} \leq \prod_{j=1}^{i} a_{j}^{b_{i} / b_{j}}\left(\bar{u}_{01}\right)^{b_{i}}=\bar{u}_{0 i}
$$

Then a solution $u^{*}$ of $(1.1)$ with $u^{*}(0)=\bar{u}_{0}$ belongs to $M$, so it satisfies

$$
\left\{\begin{array}{l}
u_{i t}^{*}-d_{i} \Delta u_{i}^{*} \leq A_{i}\left(u_{1}^{*}\right)^{\left(1+\alpha_{i}\right) / \alpha_{1}} \\
u_{i}^{*}(x, 0)=\bar{u}_{0 i}(x), \quad i=1, \ldots, m
\end{array}\right.
$$


where $A_{i}=\left(\prod_{k=1}^{m} \prod_{j=1}^{k} a_{j}^{1 / b_{j}}\right)^{\left(1+\alpha_{i}\right) / \alpha_{1}}, i=1, \ldots, m$. This yields a supersystem corresponding to (1.1) of the form

$$
\left\{\begin{array}{l}
\bar{u}_{i t}-d_{i} \Delta \bar{u}_{i}=A_{i} \bar{u}_{1}^{\left(1+\alpha_{i}\right) / \alpha_{1}}, \\
\bar{u}_{i}(x, 0)=\bar{u}_{0 i}(x),
\end{array}\right.
$$

with constants $A_{i}$ as above. Thus $u_{i}(x, t) \leq \bar{u}_{i}(x, t)$. We notice that the first equation in (5.10) is scalar, i.e.

$$
\begin{aligned}
& \bar{u}_{1 t}-d_{1} \Delta \bar{u}_{1}=A_{1} \bar{u}_{1}^{r}, \quad \text { where } r=1+1 / \alpha_{1}, \\
& \bar{u}_{1}(x, 0)=\bar{u}_{01}(x) .
\end{aligned}
$$

Let us now consider this equation. We have two separate cases when a solution of (1.1) blows up in finite time. If $p_{1}^{1} \leq 1$ (assuming that $\min _{i} \sum_{k=1}^{m} p_{k}^{i}$ $\left.=\sum_{k=1}^{m} p_{k}^{1}\right)$ then by Theorem 2 we have $\alpha_{1}=\max _{i} \alpha_{i}>0$. If $p_{1}^{1}>1$ then by Theorem 1 we can only claim that $\sum_{k=1}^{m} p_{k}^{1}-1>0$. On the other hand

$$
1=\left(p_{1}^{1}-1\right) \alpha_{1}+\sum_{k=2}^{m} p_{k}^{1} \alpha_{k} \leq\left(\sum_{k=1}^{m} p_{k}^{1}-1\right) \max _{k} \alpha_{k}
$$

so $\max _{k} \alpha_{k}=\alpha_{1} \geq\left(\sum_{k=1}^{m} p_{k}^{1}-1\right)^{-1}>0$. It follows that $r>1$.

LEMMA 5.2. Let $v(x, t)$ be a solution of

$$
\begin{array}{ll}
v_{t}-d \Delta v=A v^{r}, & x \in \mathbb{R}^{N}, t \in(0, T), \\
v(x, 0)=v_{0}(x), & x \in \mathbb{R}^{N},
\end{array}
$$

where $r>1, v_{0}(x)>0, d, A>0$. Then

$$
v(x, t) \leq C(T-t)^{-\alpha} \quad \text { with } \alpha=\frac{1}{r-1}
$$

provided that either

$$
d \Delta v_{0}+A v_{0}^{r}>0
$$

or

$$
N=1,2 \quad \text { or } \quad N \geq 3 \text { and } \alpha \geq \frac{N-2}{4} .
$$

Pr o of. Suppose that (5.13) holds. Set

$$
F=v_{t}-\delta A v^{r}
$$

where $\delta>0$ is a constant to be determined. The function $F$ satisfies

$$
F_{t}-d \Delta F=A\left(v^{r}\right)^{\prime} v_{t}-\delta A^{2}\left(v^{r}\right)^{\prime} v^{r}+\delta A d\left(v^{r}\right)^{\prime \prime}|\nabla v|^{2}
$$

and by (5.15),

$$
F_{t}-d \Delta F=A r v^{r-1} F=\delta A d r(r-1) v^{r-2}|\nabla v|^{2} .
$$

This implies, as $r>1$,

$$
F_{t}-d \Delta F-A r v^{r-1} F \geq 0 .
$$


We remark that we can choose $\delta>0$ small enough to guarantee that $F(0)>0$. Indeed, by $(5.13)$ we have $v_{t}(0) \geq c>0$, and clearly $v^{r}(0) \leq c^{\prime}$ as long as $T>0$. Then, by comparison and (5.15), (5.16), it follows that $F$ cannot be negative, i.e. there exists $\delta>0$ such that

$$
v_{t} \geq \delta A v^{r} \text {. }
$$

This is equivalent to

$$
-\frac{\partial}{\partial t}\left(\frac{v^{-r+1}}{r-1}\right) \geq \delta A
$$

or, by integration,

$$
\frac{v(x, t)^{-r+1}}{r-1} \geq \delta A(T-t)
$$

Finally, we obtain

$$
v(x, t) \leq C(T-t)^{-\alpha}, \quad \text { where } \alpha=\frac{1}{r-1}, C=((r-1) \delta A)^{-\alpha} .
$$

It remains to consider the case where (5.14) holds. Then, by assumption,

$$
N \leq 2 \quad \text { or } \quad N \geq 3 \text { and } 1<r \leq \frac{N+2}{N-2}
$$

and we can apply the relevant result proved in [GK] (cf. Theorem 3.7). Thus, our assertion follows.

Continuation of the proof of Theorem 4. Employing Lemma 5.2 in (5.11) we conclude that

$$
u_{1}(x, t) \leq \bar{u}_{1}(x, t) \leq C(T-t)^{-\alpha_{1}} .
$$

Notice that starting from $u^{*}(x, t)$ (which is also a solution of (1.1)) instead of $u(x, t)$ we can obtain the same bound:

$$
u_{1}^{*}(x, t) \leq C(T-t)^{-\alpha_{1}}
$$

Moreover, since $u^{*}(x, t) \in M$, we have

$$
u_{1}^{*} \leq\left(\prod_{j=1}^{i} a_{j}^{1 / b_{j}} u_{1}^{*}\right)^{b_{i}} \leq C_{i}(T-t)^{-\alpha_{1} b_{i}}=C_{i}(T-t)^{-\alpha_{i}} .
$$

It remains to observe that $u_{i}^{*}(x, t)$ is, by construction, a supersolution to (1.1). Therefore, the upper estimate (5.19) also holds for a solution $u(x, t)$ of (1.1). This establishes our assertion.

\section{REFERENCES}

[AHV] D. A ndreucci, M. A. Herrero and J. J. L. Velázquez, Liouville theorems and blow up behaviour in semilinear reaction diffusion systems, Ann. Inst. H. Poincaré 14 (1997), 1-53. 
[CM] G. Caristi and E. Mitidieri, Blow up estimates of positive solutions of a parabolic system, J. Differential Equations 113 (1994), 265-271.

[EH] M. Es cobedo and M. A. Herrero, Boundedness and blow up for a semilinear reaction-diffusion system, ibid. 89 (1991), 176-202.

[EL] M. E s co be do and H. A. L e vin e, Critical blow up and global existence numbers for a weakly coupled system of reaction-diffusion equations, Arch. Rational Mech. Anal. 129 (1995), 47-100.

[Fu1] H. F u j it a, On some nonexistence and nonuniqueness theorems for nonlinear parabolic equations, in: Proc. Sympos. Pure Math. 18, Amer. Math. Soc., 1970, 105-113.

[Fu2] - On the blowing up of solutions of the Cauchy problem for $u_{t}=\Delta u+u^{1+\alpha}$, J. Fac. Sci. Univ. Tokyo Sect. IA Math. 13 (1966), 109-124.

[GK] Y. Gig a and R. V. K o h n, Characterizing blow-up using similarity variables, Indiana Univ. Math. J. 36 (1987), 1-40.

[H] D. Hen ry, Geometric Theory of Semilinear Parabolic Equations, Springer, Heidelberg, 1981.

[L] G. L u, Global existence and blow-up for a class of semilinear parabolic systems: a Cauchy problem, Nonlinear Anal. 24 (1995), 1193-1206.

[LS] G. Lu and B. D. S leeman, Subsolutions and supersolutions to systems of parabolic equations with applications to generalized Fujita type systems, Math. Methods Appl. Sci. 17 (1994), 1005-1016.

[R1] J. Rencławowicz, Global existence and blow up of solutions for a completely coupled Fujita type system of reaction-diffusion equations, Appl. Math. (Warsaw) 25 (1998), 313-326.

[R2] - Global existence and blow up of solutions for a class of reaction-diffusion systems, J. Appl. Anal., to appear.

[R3] - Global existence and blow up of solutions for a weakly coupled Fujita type system of reaction-diffusion equations, ibid., to appear.

[R4] - Global existence and blow-up for a completely coupled Fujita type system, Appl. Math. (Warsaw) 27 (2000), 203-218.

Institute of Mathematics

Polish Academy of Sciences

Śniadeckich 8

00-950 Warszawa, Poland

E-mail: jr@impan.gov.pl 